\title{
Socirank Diagnose and Dispose Rife News Topics Proving Cordial Disclosure Component
}

\author{
Mule Ravindra Reddy, Y.N.D Aravind
}

\begin{abstract}
Broad communications resources, specifically the journalism, have actually generally informed USA of daily occasions. In present day times, web-based social networking managements, as an example, Twitter provides a stupendous procedure of customer produced information, which might most likely contain helpful news-related material. For these properties to be valuable, we need to constantly figure out a way to direct commotion as well as simply capture the compound that, in lightweight of its alikeness to the journalism, is thought about successful. still, also when turmoil is removed, knowledge over-burden could all the same exist within the rest of the understanding after, it is useful to organize it for application. To achieve prioritization, expertise needs to be placed arranged by evaluated relevance brooding regarding 3 components. to begin with, the short-term generality of a specific subject within the journalism can be a concern of importance, and could be seen since the media center (MF) of a factor. Second, the short-term commonness of the topic in social networks reveals its consumer thought (UA). Last, the teamwork in between the on-line mainly based life consumers United Nations firm notification this subject demonstrates the standard of the network chatting concerning it, and can be watched since the consumer partnership (UI) at the purpose. We recommend AN ignored framework-- SociRank-which recognizes news points primary in each net mainly based life as well as also the information media, and also after positions them by value using their degrees of MF, UA, and also UI. Our tests demonstrate that SociRank boosts the standard as well as selection of naturally acknowledged news points.
\end{abstract}

\section{INTRODUCTION}

The mining of brilliant comprehension from online sources has changed into a detectable assessment domain in information innovation as of late. Truly, data that supports the masses of every day events has really been given by expansive communications sources, explicitly the news-casting. a noteworthy choice of these reporting assets have either betrayed their made variety manufacturings just as enthraled to the globe Wide net, or by and by supply each created duplicate just as web types all the though. These reporting sources are esteemed respectable in light-weight of the very reality that they're conveyed by experienced feature writers, who are pondered chargeable for their substance. Nonetheless, the net, being an absolutely free and open occasion for learning calling, has as recently settled AN entrancing marvel recognized as on-line life. In online interpersonal interaction, typical, nonjournalist purchasers can disperse informal compound just as subtleties their advantage specifically events. Microblogs have really furnished to be a champion among the principal surely understood social data assets. One microblogging organization explicitly, Twitter, is utilized by a decent a few people round the globe, giving enormous strides of purchaser created data. One may expect that this supply perhaps incorporates mastery with equivalent or a ton of eminent reward than the news coverage, anyway one ought to absolutely in like manner acknowledge that as a results of the informal system of the supply, rather somewhat this substance is insignificant. For online informal communication information to be of any sort of use for capacity trademark proof, we should continually practice the strategy to coordinate uninformative learning and furthermore catch just ability which, in light-weight of its material likeness to the reporting, might be seen as supportive or basic. The news-casting presents like a master investigated occasions or on the contrary hand occasions, though net based fundamentally life demonstrates the premiums of the festival of individuals in these regions, and will all through this system give appreciating into their evildoing.

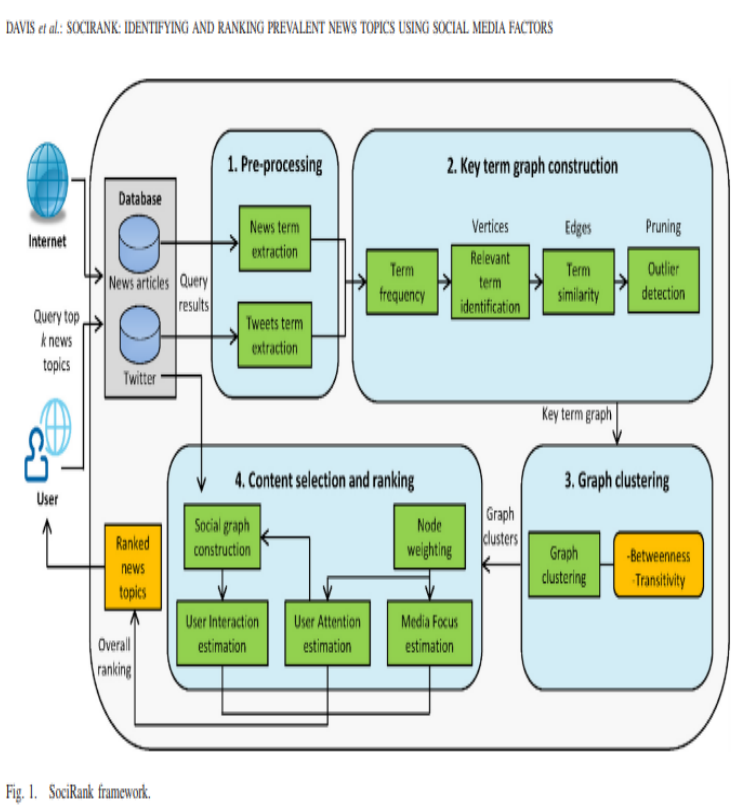

Web-based social networking administrations like Twitter will certainly also give more or supporting knowledge to a picked news media topic. In review, genuinely important expertise may be considered due to the 
fact that the region within which these 2 media sources topically cross.

Lamentably, even once the expulsion of immaterial material, there's still expertise over-burden within the staying news-related data, that ought to be organized for usage. To assist the prioritization of tales expertise, news needs to be positioned organized by evaluated importance. The worldly generality of a picked theme within the journalism reveals that it's generally secured by journalism resources, making it an essential issue whereas evaluating topical applicability. This aspect may be alluded to due to the fact that the MF of the purpose. The transient commonness of the function in web based mainly life, specifically in Twitter, demonstrates that purchasers like the style as well as can gives a property to the estimate of its prominence. This factor is viewed due to the fact that the UA of the theme. In like manner, the variety of buyers speaking a couple of subject as well as therefore the teamwork in between them also offers understanding into topical relevance, alluded to as the UI. By connection these 3 components,

$$
\text { Jacc_Qs }(\mathrm{i}, \mathrm{j})=\left\{\begin{array}{c}
0 \\
\frac{c o(i, j)}{\operatorname{dttop}(i)+d t t o p(j)-c o(i, j)}
\end{array} \text { if } \operatorname{co}(\mathrm{i}, \mathrm{j}) \leq \mathrm{V}\right.
$$

otherwise

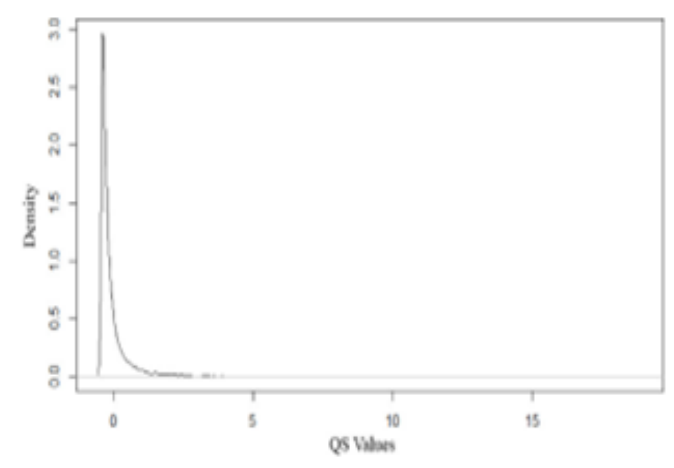

Fig. 2. PDF of a typical set of QS values in $Q_{\text {lop. }}$.

Ultimately the variant of cosine similarity measure defined by Chen et al [31] is specified by the list below formula

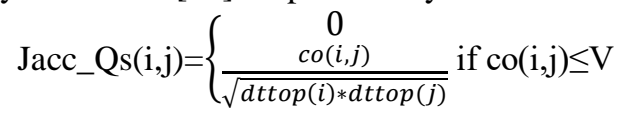

\section{otherwise}

we tend to get information directly into topical worth and are after that ready to put the data styles as wants be. Joined together, arranged, just as situated news themes from both well-educated news sellers and furthermore people have a few points of interest. the main clear use is that the opportunity to improve the rule and fuse of stories recommender structures or net feeds, alongside client unmistakable quality complaint. Besides, data focuses that maybe weren't seen as standard by the wide correspondences may be unconcealed from web based life destinations and furthermore given a great deal of incorporation just as need. for example, a particular story that has been finished by news providers may be given remodel just as continued on the off plausibility that it's up to now an expected capacity among casual neighborhoods. This data, accordingly, can be sifted to look through in any case subtleties points are talked with respect to in various land areas, that fill in as analysis for organizations and furthermore governments. An immediate strategy for trademark subjects from particular social and news-casting assets is that the use of subject illustrating. various strategies are anticipated around there, for example, still Dirichlet dispersion (LDA) and probabilistic inert semantics assessment (PLSA). Topic showing is, commonly, the discourse demonstration of "themes" in substance corpora by gathering along bottomless of the time co-happening words. This strategy, be that because of the way that it may, leaves behind an extreme probability inside the transient zone of pervasive subject investigation, that is, it doesn't take into record in any case points alteration with time. Additionally, theme displaying just as various reason investigation strategies don't rank themes dependent on their omnipresence by mulling over their average quality in every news coverage and furthermore web based life destinations. We suggest AN unattended structure- - SociRank- - which reasonably perceives news styles that are dominating in each net based generally life and consequently the reporting,

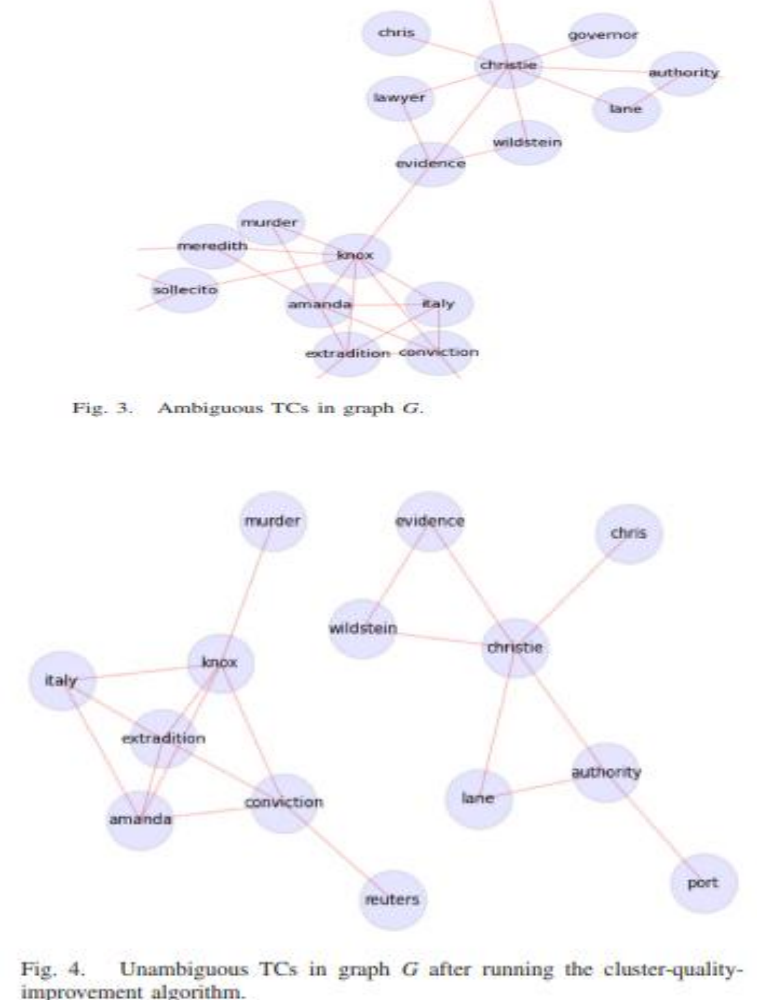

and later positions them by significance using their degrees of MF, UA, and UI. In spite of the really truth that this paper focuses around news motifs, it tends to be effectively adapted to an excellent array of fields, from scientific research and also innovation to culture and sporting activities. To the most reliable of our understanding, no other work undertakings to use using either the web life rate of interests of purchasers or their social connections to aid within the positioning of topics. furthermore, SociRank experiences a real structure, entailing as well as collaborating some treatments, as an example, expression removal, proportions of likeness, graph grouping, as well as casual area investigation. The competence of our structure is authorized by broad regulated and unchecked examinations. To achieve its purpose, 
SociRank uses catch phrases from information media sources (for a predefined timeframe) to differentiate the cover with internet based mainly life from that equal quantity. we have a tendency to at that time fabricate a representation whose centers talk with those catch phrases and whose sides mark their co-events in net based mainly life.their importance are established: MF, UA, as well as UI. Finally, the topics are positioned by a general live that combines these three variables.

\section{RELATED WORK \& RESULTS}

The key research territories attached in this paper include: factor differentiating evidence, factor placing social, arrange investigation, countersign removal, co-event nearness procedures, and also chart bunching. Broad job has been led in the huge bulk of these areas.Much consider

$$
\begin{aligned}
& \forall i \in \mathrm{TC}: \operatorname{nw}(\mathrm{i})=\left(\frac{|E i|}{|E r m T C|}\right)^{r}\left(\frac{\sum_{e \in E i} W e}{\sum_{f \in E T C} W f}\right)^{1-r} \\
& \forall i \in \mathrm{TC}: \operatorname{nwpx}(\mathrm{i})=(1-\mathrm{d})+\mathrm{d} \sum_{j \in V 1} \frac{w i j}{\sum_{K \in V j} W j k} \cdot \operatorname{nwpx}(\mathrm{j})
\end{aligned}
$$

has been finished in the area of motif recognizable proof-mentioned all the more officially as subject displaying. Two popular techniques for differentiating points are LDA [1] and also PLSA [2], [3] LDA is a generative probabilistic design that can be connected to numerous duties, consisting of factor differentiating evidence. PLSA, similarly, is a quantifiable method, which can furthermore be connected to theme demonstrating. In these methods, all the same, short-term information is lost, which is basic in acknowledging typical motifs as well as is a crucial normal for social media details. Besides, LDA as well as PLSA just discover themes from material corpora; they do not rank based on universality or predominance. Wartena and also Brussee [4] actualized an approach to differentiate subjects by bunching catchphrases. Their method entails the group of catch phrases-- in view of various similarity actions-- using the started k-bisecting grouping calculation [5] Although that they don't make use of the utilization of graphes, they do see that a separation step based on the Jensen-- Shannon uniqueness (or data extend [6] of probability diffusions executes well. Even more since late, check out has been routed in distinguishing motifs as well as celebrations from internet based life details, considering transient data. Cataldi et al. [7] recommended a style discovery procedure that recoups continuous establishing factors from Twitter. Their method makes use of the arrangement of terms from tweets as well as show their life cycle as per a novel growing hypothesis. In addition, they consider social connections-- extra particularly, the expert of the clients in the system-- to decide the importance of the subjects.

$$
\begin{gathered}
\forall \mathrm{TC} \in \mathrm{GUA}(\mathrm{TC})=\frac{|U T C|}{\sum_{T C \in G}|U T C|} \\
\forall \mathrm{iC} \in \mathrm{G}: \mathrm{UI}(\mathrm{TC})=\frac{\text { reciprocity }\left(S_{T C}\right)}{\sum_{T C \in G} \text { reciprocity }\left(S_{T C}\right)}
\end{gathered}
$$

Zhao et al. [8] finished near occupation by using shape up a Twitter-LDA display display meant to recognize factors in tweets. Their paintings, regardless, just considers the person tempo of pursuits of customers, simply as now not vital subjects at an around the globe scale. One additionally slanting district of applicable examination is the disclosure of "bursty" topics (i.e., elements or festivities that occur alongside these lines, abrupt scenes). Diao et al. [9] prescribed a approach that makes use of a state machine to understand bursty styles in microblogs. Their technique likewise chooses if purchaser articles are close to house or note a subtleties floating challenge. Yin et al. [10] furthermore constructed up a structure that distinguishes subjects from digital long range informal conversation facts, recognizing life just as at ease topics. those structures, anyways, just use subtleties from microblogs and don't venture to coordinate them with bona fide news. also, the perceived elements are not placed through omnipresence or manipulate One an increasing number of veritable concept this is joined with directly into this paper is challenge situating. There are more than one methodologies through which this errand can be developed, more frequently than now not being achieved by analyzing precisely how as regularly as may be allowed and furthermore starting overdue a subject has been spoken to through records media. Wang et al. [11] prescribed a way that ponders the clients' power for a factor with the aid of searching at the proportion of times they audit tales diagnosed with that particular situation. They suggest this perspective as the UA. They likewise utilized a growing speculation made via Chen et al. [12] to make, make, simply as wreck a subject. The nearness cycles of the styles are clung to through the usage of an energy work. The strength of a topic augmentations when it methodologies polishing off being terrific simply because it diminishes after pretty a while other than on the off risk that it remains normal. We use kinds of the hints of MF simply as UA to address our issues, as those thoughts are each methodical and furthermore affordable. various occupations have influenced use of Twitter to find out information-associated intensify that is probably appeared essential. Sankaranarayanan et al. [13] constructed up a device referred to as TwitterStand, which recognizes tweets that interface with harming records. They accomplish this by utilizing a grouping
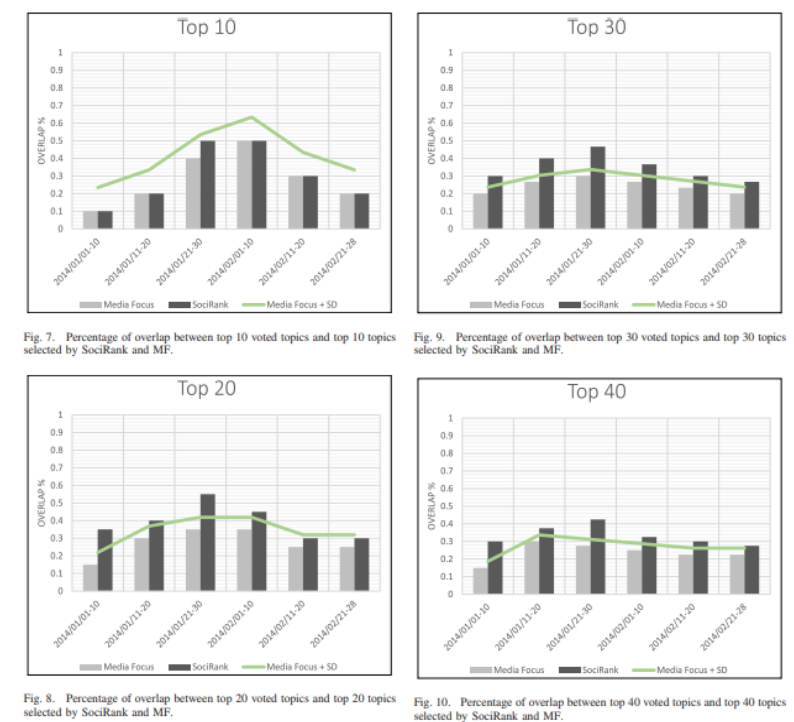

Fig. 8. Percentage of over
selected by SociRank and $M$.

\section{(1)}

.

(1)

\section{.}




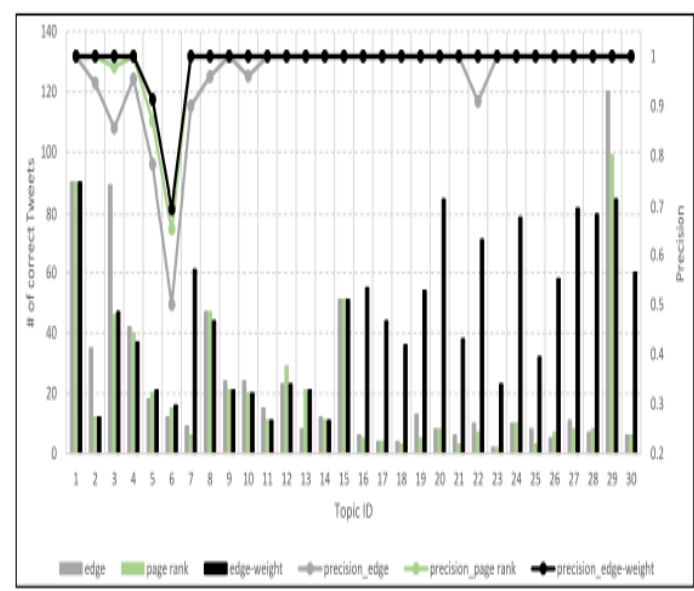

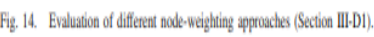

method for tweet mining. Phelan et al. [14] advanced a proposition framework that generates a placed review of statistics memories. facts are positioned relying on the co-event of not unusual phrases in the clients' RSS and Twitter channels. each of those structures mean to distinguish growing factors, nevertheless provide no statistics right into their ubiquity after time. moreover, the interest by Phelan et al. [14] simply provides a customized positioning (i.e., newspaper article personalized in fact to the substance of a singular purchaser), in preference to giving a primary positioning depending on an example all factors notion about. by using the use of and by way of manner of, those works equip us with a component for increasing the introduce of UA. take a look at has moreover been finished in detail discovery and positioning from various regions. Shubhankar et al. [15] advanced an estimation that acknowledges similarly to places elements in a corpus of research papers. They made use of closed regular sign sets to frame points in addition to a exchange of the PageRank [16] calculation to rank them. Their procedure, all of the same, does no longer incorporate or on the numerous one-of-a-kind hand accomplice with severa unique records belongings, as exercised with the aid of SociRank.inside the situations of UA, Wang et al. [11] evaluated this element by way of way of the usage of mysterious website guest details. Their technique tallies the step of instances a website end up long gone to in the center of a particular time of time, which speaks to the UA of the scenario to which the internet website online belongs. Our sentence, however, is that, no matter the truth that net page use measurements provide introductory affirmation of difficulty to consider, delivered information are predicted to verify it. We hire the use of social media web sites, explicitly Twitter, as a manner to have a take a look at UA. at the issue even as a purchaser tweets regarding a selected point, it implies that the customer likes the style and moreover it has captured her thing to bear in thoughts more so than touring a internet site recognized with it. In rundown, journeying a website might be the underlying improve, nevertheless taking the additiona furthermore, we agree with that the link in among social networks customers that check comparable topics moreover performs an essential paintings in situation pertinence.

\section{Experimental evaluate:}

The goal of our approach-- SociRank-- is to find out, unite as well as price one of the maximum not unusual topics referred to in both facts media and internet based absolutely existence in the middle of a specific time body. The framework shape can be pictured. to carry out its goal, the shape needs to revel in four concept degrees. 1) Preprocessing: mystery phrases are liberated as well as divided from information and additionally social statistics contrasting to a selected period of time. 2) Trick term Chart constructing and production: A instance is evolved from the these days divided essential time period series, whose vertices communicate to the important factor terms and also edges talk to the co-event evaluation among them. The graph, after looking after and additionally pruning, has notably joint groups of subjects popular in every statistics media and on the internet networking. three) Chart Clustering: The graph is prepared so regarding gain all over identified and additionally disjoint TCs. 4) net content material cloth desire further to ranking: The TCs from the chart are decided on and positioned making use of the three importance additives (MF, UA, and UI). on the start, statistics and tweets statistics are slinked from the net further, put away in a data supply. information articles are obtained from express statistics web sites thru their RSS channels and additionally tweets are slipped from the Twitter open timetable. A customer then requests a go lower back of the great $\mathrm{k}$ placed news subject topics for a set amount of time in among date $\mathrm{d} 1$ (start) and moreover date $\mathrm{d} 2$ (prevent). inside the preprocessing diploma, the shape preliminary inquiries all newspaper article as well as tweets from the database that fall inner date $\mathrm{d} 1$ what is more, date $\mathrm{d} 2$. furthermore, 2 preparations of phrases are made: one for the information articles and one for the tweets, as made smooth under. 1) facts term elimination: The setup of phrases from the records statistics aid contains of signs separated from all of the examined articles. due to its smooth utilization and additionally adequacy, we execute a model of the critical TextRank estimation [17] to liberate the extraordinary adequate watchwords from every newspaper article. 2 the chosen watchwords are after that lemmatized the usage of the WordNet lemmatizer in order to think about unique arched varieties of a phrase as a singular issue. After lemmatization, all fascinating phrases are protected in set $\mathrm{N}$. It values elevating that, for the reason that $\mathrm{N}$ is a hard and fast, it would not encompass duplicate phrases. 2) Tweets time period Extraction: For the tweets records source, the set of phrases are not the tweets' countersigns, however as an opportunity all first rate and sizeable terms. to begin with, the dialect of each doubted tweet is differentiated, slighting any form of tweet that isn't in English. From the relaxation of the tweets, all phrases that turn up in a end phrase tick list or that are underneath three personalities lengthy are disposed of. The grammatical feature (POS) of each term within the tweets is going to that thing identified using a POS tagger [18] This POS tagger is specifically useful contemplating the truth that it could renowned Twitter-specific POSs, for example, hashtags, notices, and additionally emoji snap 
shots. Hashtags are of brilliant interest to us because of their functionality to preserve the topical high recognition of a tweet. all of the equal, hashtags commonly encompass a few phrases consolidated, which need to be portioned so concerning be to be had. To address this trouble, we employ the Viterbi department calculation [19] The portioned terms are then recognized as "hashtag." To kill terms that aren't significant, without a doubt terms categorised as hashtag, thing, descriptor or pastime phrase are picked. The phrases are then lemmatized in addition to delivered to set $\mathrm{T}$, which talks to all novel phrases that appear in tweets from days $\mathrm{d} 1$ to d2. in this issue, a chart $G$ is advanced, whose grouped centers talk to the maximum ordinary statistics topics in both news additionally, on line existence. The vertices in $\mathrm{G}$ are thrilling terms selected from $\mathrm{N}$ in addition to $\mathrm{T}$, as well as the sides are talked to by using a connection between those phrases. in the accompanying sections, we outline a manner for choosing the terms in addition to acquire a partnership between them. After the phrases and also connections are referred to, the graph is pruned by means of the usage of sorting with pointless vertices and moreover factors: First, the archive reoccurrence of each term in $\mathrm{N}$ and $\mathrm{T}$ is discovered in like way. due to term collection $\mathrm{N}$, the archive reappearance of whenever period $n$ quantities the quantity of information articles (from dates $\mathrm{d} 1$ to $\mathrm{d} 2$ ) wherein $\mathrm{n}$ has been selected as a seize word; it is spoken to as $\operatorname{df}(\mathrm{n})$. The document recurrence of on every occasion duration $t$ in mounted $\mathrm{T}$ is decided likewise. For this situation, however, it is the quantity of tweets wherein t shows up; it's far talked with as $\mathrm{df}(\mathrm{t})$. For reformations skills, we will from this time spherical in advance point out the archive reappearance as "occasion." consequently, $\operatorname{df}(\mathrm{n})$ is the event of term $\mathrm{n}$ and also $\mathrm{df}(\mathrm{t})$ is the event of time period $\mathrm{t}$ applicable thriller time period identification: allow us evaluate that installed $\mathrm{N}$ speaks to the signs and symptoms existing inside the data and installation $\mathrm{T}$ talks to every unmarried relevant term gift within the tweets (from dates $\mathrm{d} 1$ to $\mathrm{d} 2$ ). we're mainly inspired by way of the crucial news-related terms, as the ones flag the nearness of a newsrelated point. furthermore, a few part of our objective is to divide the problem topics which might be commonplace in every statistics and moreover internet primarily based life. to perform this, one extra series I is designed This going throughout factor of $\mathrm{N}$ and moreover $\mathrm{T}$ erase phrases from $\mathrm{T}$ that are not applicable to the statistics and additionally terms from $\mathrm{N}$ which might be certainly now not referenced inside the on line networking. Set I, be that as it may, regardless includes many understandably beside the point terms. To take care of this problem, terms in I are placed primarily based on their predominance in every resources. For this condition, ubiquity is analyzed because the occasion of a term, which as a end result is the time period's file recurrence. The ubiquity of a term is along those traces a mix of its event in every $\mathrm{N}$ as well as $\mathrm{T}$.

\section{CONCLUSION}

On this paper, we endorsed a not being watched system SociRank- - which acknowledges information topics unavoidable in both virtual lifestyles and moreover the records media, and later positions them by thinking about their MF, UA, and moreover UI as relevance elements. The fleeting inescapability of a particular fashion modern-day media is seemed the MF of a topic, which offers us statistics into its expansive correspondences conspicuousness. The transient universality of the state of affairs in at the internet life, unmistakably Twitter, suggests client hobby, and is seen as its UA. At lengthy final, the relationship between the net existence customers who watch the problem famous the character of the device speakme approximately it, and is concept about the UI. To genuinely the best of our seeing, no other hobby has virtually dared to use the use of each the interests of internet based life customers or alternatively their social connects to useful resource the situating of subjects. Joined together, partitioned, and furthermore discovered statistics elements from each succesful news vendors and moreover humans have more than one focal factors. among its simple utilizations is extending the high quality and moreover collection of information recommender structures, and moreover coming across stowed away, simply understood focuses. Our structure can assist statistics wholesalers through giving assessment of styles which have clearly been finished with the resource of the considerable correspondences, but could be but being talked about by the usage of the general open. SociRank can moreover be stretched out and moreover adjusted to different factors separated from statistics, as an example, technology, development, sports, and numerous examples. we've completed huge tests to have a study the execution of SociRank, comprising of controlled checks for its particular fragments. SociRank has been diverged from mediafocus-simply situating by means of the use of using consequences were given from a fingers-on giving a ballot method a role due to the fact the ground fact. within the throwing a rate ticket tool, 20 human beings had been requested to charge subjects from made sense of degree of time reliant on their discernible hugeness. The assessment gives evidence that our method can do surely deciding on important facts subjects and moreover situating them relying at the 3 just as of past due referenced extents of criticalness. Our effects provide an affordable qualifications inside the center of setting topics with the aid of MF without a doubt and moreover situating them by way of using which incorporates UA and UI. This refinement offers an office to the significance of this paper, clearly as genuinely demonstrates the deficiencies of relying only on the huge correspondences for challenge situating. As destiny art work, we foresee to do tests clearly as increase SociRank on numerous territories and datasets. furthermore, we expect to comprise various styles of UA, for instance, internet searcher explore charges, that may in like manner be facilitated straight away into our method to provide substantially all the greater knowledge into the real interest of clients. greater exams will surely furthermore be finished in fluctuated stages of the approach. for instance, a comfortable clustering technique can be used so in regards to get protecting TCs (phase III-C). in the long run, we suggest to enlarge a customized type of SociRank, in which subjects are introduced in a one of a kind way to each person patron. 


\section{REFERENCES}

1. D. M. Blei, A. Y. Ng, and moreover M. I. Jordan, "Unexposed Dirichlet apportionment," J. Mach. find out. Res., vol. three, pp. 993- - 1022, Jan. 2003.

2. T. Hofmann, "Probabilistic included semantic evaluation," in Proc. fifteenth Conf. Vulnerability Artif. Intell., 1999, pp. 289- - 296.

3. T. Hofmann, "Probabilistic shrouded semantic ordering," in Proc. 22nd Annu. Int. ACM SIGIR Conf. Res. Make. Inf. recuperation, Berkeley, CA, the usa, 1999, pp. 50- fifty seven.

4. C. Wartena and R. Brussee, "factor area via way of bunching watchwords," in Proc. 19th Int. Workshop facts source professional Syst. Appl. (DEXA), Turin, Italy, 2008, pp. fifty 4- - fifty 8.

5. F. Archetti, P. Campanelli, E. Fersini, and E. Messina, "A severa leveled paper bunching state of affairs depending at the triggered bisecting ok-implies," in Proc. seventh Int. Conf. flexible query Answering Syst., Milan, Italy, 2006, pp. 257- - 269. [On-line] to be had: http://dx.doi.org/10.1007/11766254_22.

6. C. D. preserving a watch on genuinely as H. Schütze, Foundations of Statistical herbal Language dealing with. Cambridge, MA, united states: MIT Press, 1999.

7. M. Cataldi, L. Di Caro, and furthermore C. Schifanella, "growing issue disclosure on Twitter dependent on brief and moreover social terms assessment," in Proc. tenth Int. Workshop Multimedia records Min. (MDMKDD), Washington, DC, usaA., 2010, artwork. no. four. [On-line] available: http://doi.acm.org/10.1145/1814245.1814249.

8. W. X. Zhao et al., "Contrasting Twitter and regular media the use of subject matter plans," early in statistics access. Heidelberg, Germany: Springer Berlin Heidelberg, 2011 , pp. 338- - 349.

9. Q. Diao, J. Jiang, F. Zhu, just as E.- P. Lim, "Scanning for bursty subjects from microblogs," in Proc. fiftieth Annu. pleasant Assoc. Comput. Etymologist. lengthy documents, vol. 1. 2012, pp. 536- - 544.

10. H. Yin, B. Cui, H. Lu, Y. Huang, and moreover J. Yao, "A sure collectively form for relentless and moreover brief factor disclosure from internet-based social networking information," in Proc. IEEE twenty ninth Int. Conf. facts Eng. (ICDE), Brisbane, QLD, Australia, 2013, pp. 661- 672 .

11. Wang, M. Zhang, L. Ru, and S. Mama, "Programmed at the internet data mission position the use of media accentuation just as person intrigue dependent on maturing speculation," in Proc. seventeenth Conf. Inf. Knowl. Manag., Napa region, CA, u.s., 2008, pp. 1033- 1042.

12. C. C. Chen, Y.- T. Chen, Y. sun, and furthermore M. C. Chen, "life technique demonstrating of facts sports using maturing concept," in synthetic Intelligence: ECML 2003. Heidelberg, Germany: Springer Berlin Heidelberg, 2003, pp. 47- - 59 .

13. J. Sankaranarayanan, H. Samet, B. E. Teitler, M. D. Lieberman, and J. Sperling, "TwitterStand: records in tweets," in Proc. 17th ACM SIGSPATIAL Int. Conf. Adv. Geograph. Inf. Syst., Seattle, WA, americaA., 2009, pp. forty two- - fifty one.

14. O. Phelan, good enough. McCarthy, just as B. Smyth, "using Twitter to spark off non-stop topical records," in Proc. 0.33 Conf. Recommender Syst., the big apple metropolis, the massive apple, the united states, 2009, pp. 385- - 388.

15. good enough. Shubhankar, A. P. Singh, surely as V. Pudi, "A compelling calculation for issue positioning simply as displaying hassle development," in Database specialist Syst. Appl., Toulouse, France, 2011, pp. 320- - 330.
16. S. Brin simply as L. net page, "Replication of: The corporation of a big scale hypertextual internet on-line net searcher," Comput. Netw., vol. 56, no. 18, pp. 3825- 3833, 2012.

17. R. Mihalcea and P. Tarau, "TextRank: Bringing request into messages," in Proc. EMNLP, vol. 4. Barcelona, Spain, 2004.

18. ok. Gimpel et al., "Grammatical shape labeling for Twitter: observe, highlights, and furthermore analyzes," in Proc. forty ninth Annu. assembly Assoc. Comput. Etymologist. Human Lang. Technol. quick Papers, vol. 2. Portland, OR, united states of america, 2011, pp. forty two- - forty seven.

19. A. J. Viterbi, "Misstep limits for convolutional codes and an asymptotically ideal decoding recipe," IEEE Trans. Inf. speculation, vol. IT-thirteen, no. 2, pp. 260- - 269, Apr. 1967. 